\title{
LE SPECKLE POUR MESURER la maturité des fruits climactériques
}

Marie ABBOUD Guy LE BRUN

Laboratoire de biophotonique, UR TVA, Faculté des sciences, Université Saint-Joseph de Beyrouth, Liban marie.abboud@usj.edu.lb,

2Laboratoire de Spectrométrie et Optique Laser, Université de Bretagne Occidentale, France guy.lebrun@univ-brest.fr
Plusieurs techniques sont développées pour évaluer la maturité des fruits climactériques et non climactériques. Elles sont de diverses natures : biochimiques, optiques, acoustiques, mécaniques et sont basées sur l'étude de leurs données physiologiques et leurs propriétés mécaniques, élastiques et visco-élastiques. L'évolution des recherches actuelles porte un intérêt croissant aux méthodes optiques noninvasives comme applications potentielles dans la prévision de la maturité et la durée de conservation des fruits et légumes.

les exigences minimales de qualité et de maturité des fruits et légumes frais, ainsi que les tolérances admises dans l'appréciation du respect de ces exigences y sont détaillées. Au niveau des exigences qualitatives, les fruits et légumes doivent être intacts, sains, propres, pratiquement exempts de corps étrangers visibles, de parasites ou d'altérations de la pulpe dues à des parasites, et de toute odeur ou saveur étrangères. Les produits doivent être dans un état leur permettant de supporter le transport et la manutention et d'arriver dans un état satisfaisant au lieu de destination. Quant aux exigences minimales en matière de maturité, les produits doivent être suffisamment développés afin que l'état de leur maturité permette la poursuite du processus de maturation jusqu'à ce qu'ils atteignent un degré de maturité suffisant. En pratique et afin de préserver la qualité marchande des fruits après récolte, deux solutions sont principalement mises en place. Tout d'abord, comme la maturation peut se poursuivre après récolte, le fruit doit être cueilli avant le début de la crise climactérique (voir encart). Il doit ensuite être conservé dans des conditions d'atmosphère contrôlée limitant la production d'éthylène et l'augmentation de la respiration, ralentissant ainsi sa maturation.

Parmi les techniques actuelles permettant d'évaluer la maturité ou la durée de conservation des fruits et légumes, il y a un intérêt croissant pour les méthodes optiques non-invasives. définitions des normes de commercialisation pour les fruits et légumes,
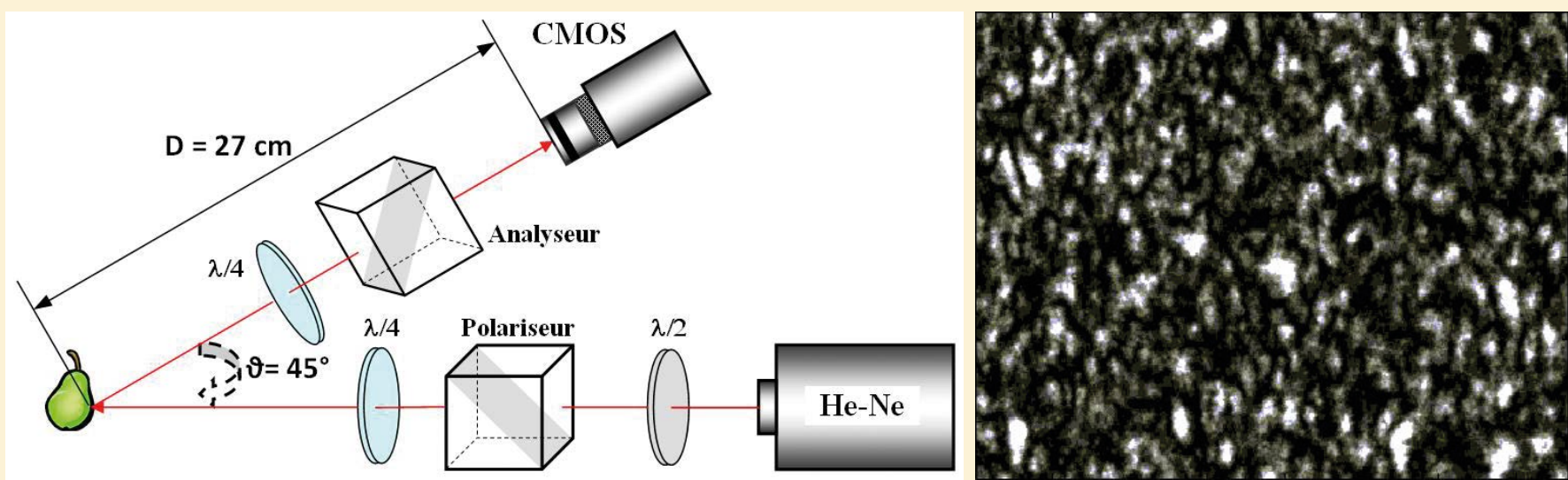

À gauche: schéma du dispositif expérimental utilisé pour les mesures de la maturité des fruits par analyse du champ de speckle couplé à des mesures de polarimétrie. À droite: exemple d'image de speckle. D est la distance entre le fruit et la caméra. $\lambda / 4$ et $\lambda / 2$ sont respectivement une lame quart $\mathrm{d}$ 'onde et demi-onde 

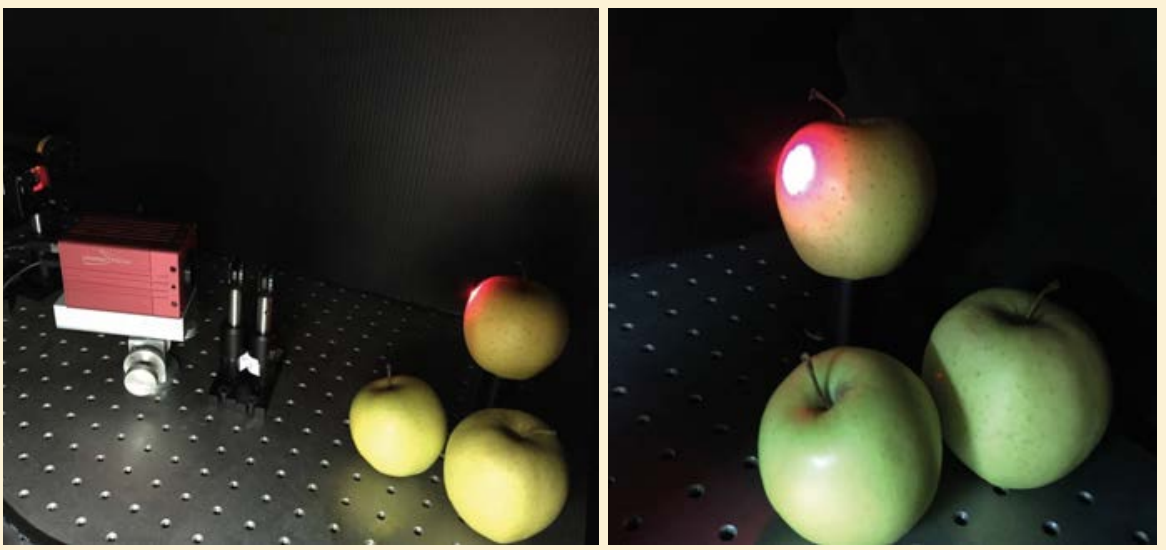

À gauche: photo du dispositif expérimental utilisé pour les mesures de la maturité des fruits par analyse du champ de speckle couplé à des mesures de polarimétrie.

À droite: photo de la tache de diffusion sur une pomme.

\section{Le speckle en quelques mots}

Le speckle, en tant que technique d'imagerie optique, utilise les propriétés de cohérence de la lumière. À cause des phénomènes de diffusion, la propagation d'une onde lumineuse très monochromatique dans un milieu biologique épais (plusieurs $\mathrm{cm}$ ) se fait selon une multitude de chemins possibles; cela signifie, en termes de photon, que celui-ci perd complètement la «mémoire» de ses caractéristiques en termes de direction et de polarisation et que la phase au cours de cette propagation subit de très fortes variations aléatoires. Cependant, les ondelettes qui vont émerger peuvent interférer, car la cohérence de la source choisie est suffisante. Dans ces milieux, les chemins parcourus par la lumière peuvent aller jusqu'à dix fois l'épaisseur traversée et donc la source lumineuse utilisée doit avoir une longueur de cohérence relativement grande.

La distribution spatiale du champ électromagnétique à la sortie du milieu biologique est aléatoire en amplitude et en phase; elle est qualifiée de granularité laser, ou encore de speckle. En effet, en chaque point de la face de sortie du milieu traversé par la lumière, le champ de la lumière est la résultante d'interférences entre un grand nombre de petites contributions indépendantes, suite aux différents chemins de propagation possibles dans le milieu.

Plusieurs paramètres peuvent être extraits à partir des images de speckle permettant une analyse spatio-temporelle de l'activité du milieu comme la taille du grain de speckle, le degré de polarisation de la lumière diffusée, la corrélation spatio-temporelle et encore la matrice temporal history speckle pattern (THSP).

\section{Speckle et fruits}

Estimer précisément le stade de maturité des fruits s'avère être une tâche complexe. Le développement de méthodes non-invasives, non destructives et instantanées constitue à l'heure actuelle un sujet de recherche et d'intérêt. Le but est d'identifier le meilleur jour pour cueillir le fruit et d'être également capable de suivre sa maturation une fois cueilli s'il est climactérique afin de prédire sa durée de conservation. La méthode considérée dans nos travaux consiste à étudier le champ de speckle généré par le fruit pour pouvoir accéder à ses propriétés physiologiques internes. En effet, les fruits montrent une activité de speckle liée à plusieurs paramètres tels la maturité, la turgescence, les dommages, la sénescence et la variation des propriétés rhéologiques.

Des mesures par analyse du champ de speckle couplées à des mesures de polarimétrie ont été effectuées sur des poires vertes, de type Conférence, et des pommes blanches, de type Golden, en parallèle avec des analyses biochimiques destructives (mesures du taux de pigmentation et du taux de glucose), des mesures de pénétrométrie, des mesures de l'intensité de fluorescence de

\section{STEMMER ${ }^{\circledR}$}

I MAG ING

\section{Vision Industrielle hyperspectrale}

Les systèmes de vision hyperspectrale de STEMMER IMAGING utilisent une plateforme de traitement de données générique développée par Perception Park. Dotée d'une interface de configuration intuitive, cette plateforme facilite les manipulations. Les méthodes scientifiques de l'analyse hyperspectrale sont dorénavant accessibles pour des applications industrielles.
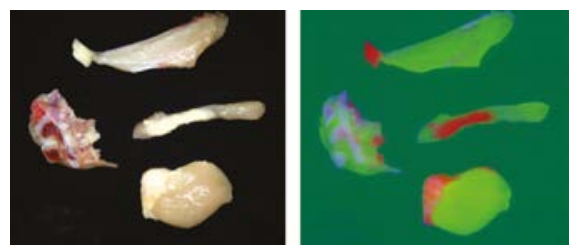

L'élément central de cette technologie réside dans l'extraction d'images bidimensionnelles, les Chemical Color Images, à partir de données hyperspectrales multidimensionnelles complexes.

Le système Perception Park permet de configurer simplement chaque application nouvelle ou d'adapter les applications existantes avec un investissement faible en termes de développement.

Dans l'industrie de transformation alimentaire par exemple, cette technologie est particulièrement appréciée.

La Vision Industrielle hyperspectrale ne se limite pas au secteur de l'alimentation, les principales industries utilisatrices sont actuellement l'industrie minière, l'industrie pharmaceutique, l'industrie du bois ainsi que le domaine du recyclage notamment pour le tri des éléments en polyéthylène $(\mathrm{PE})$ et polypropylène (PP) en fonction de leur composition chimique...grâce à cette technique, des solutions fiables à des problèmes jusqu'ici insolubles sont désormais envisageables.

\section{CONTACT}

STEMMER IMAGING S.A.S.

23 bis, rue Edouard Nieuport

92150 Suresnes, France

Tél. : +33145069560 
la chlorophylle et des mesures de la quantité d'éthylène émis par les fruits.

Ces mesures ont montré que le speckle permet d'explorer les paramètres de diffusion dans le fruit et de retrouver le pic climactérique caractérisant le pic de maturité des fruits. En effet, comme illustré à la figure 3 , l'évolution de la quantité d'éthylène dégagée avec les jours de maturation montre qu'un maximum d'éthylène dégagé, indiquant le pic climactérique, est observé au jour 8 après cueillette des pommes. À partir de ce jour, la quantité d'éthylène diminue et les fruits entrent en phase de sénescence. L'évolution de la taille des grains de speckle des pommes est également présentée à la figure 3. Le paramètre
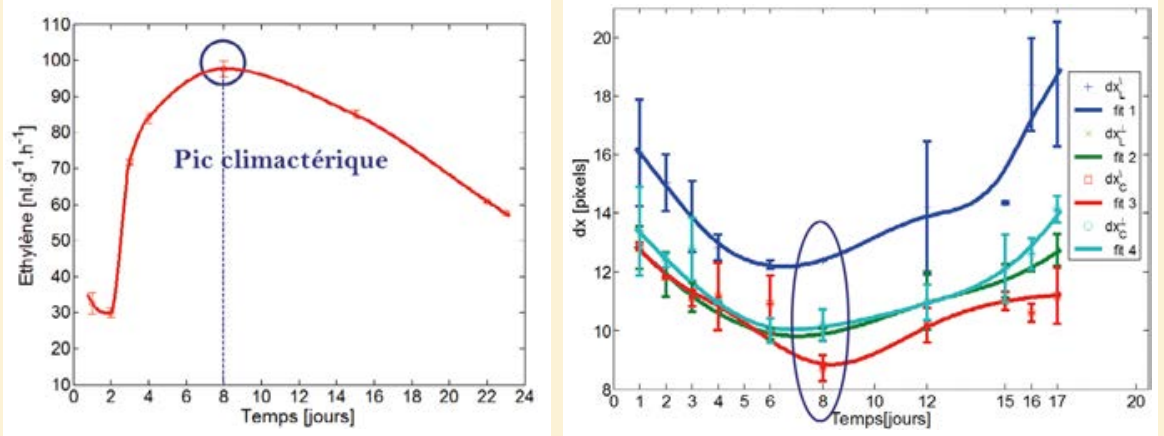

Pendant la maturation des pommes Golden cueillies au jour 0 et conservées à température ambiante de $20^{\circ} \mathrm{C}$ : variation de la quantité d'éthylène dégagée (à gauche); variation de la taille des grains de speckle (à droite).

$d x$ (taille des grains de speckle) diminue de 4 pixels en moyenne pour atteindre un minimum au jour 8 , au-delà duquel la taille de grain augmente.
Cette observation est valable pour les configurations en polarisation linéaire, avec une taille de grains en configuration parallèle $d x{ }_{L}$ supérieure à celle en

\section{Fruits climactériques}

La maturation des fruits correspond à une séquence de changements biochimiques et physiologiques conduisant à l'état de maturité et conférant au fruit ses caractéristiques organoleptiques telles que le goût, les arômes, la couleur, la texture, qui rendent le fruit commercialisable et comestible. Bien que la plupart des modifications au cours de la maturation soient communes à la plupart des fruits, leur capacité à produire de l'éthylène durant cette phase de leur vie les répartit en deux catégories: les fruits climactériques, caractérisés par un pic de respiration et de production d'éthylène, et les fruits non climactériques qui ne montrent pas un changement de production d'éthylène durant la maturation.

Les fruits non climactériques, comme l'ananas, les raisins, ou encore les fraises, ne peuvent pas être cueillis avant qu'ils soient mûrs. En effet, comme ces fruits ne présentent aucune autonomie de maturation après récolte, les critères de leur récolte sont analogues aux critères de qualité gustative reposant sur une estimation de leur niveau de développement et de leur stade de maturité. Deux principaux types de critères sont utilisés: les critères visuels tels la forme, le calibre, la couleur; et les critères analytiques tels la fermeté, la teneur en sucre et l'acidité.

Les fruits climactériques, comme les pommes, les poires, les bananes, les melons, les pêches, les tomates se distinguent par une phase climactérique et des caractéristiques physiologiques assez particulières. Ces fruits doivent être récoltés après avoir atteint un niveau de développement physiologique, donc de maturité, suffisant. Ce n'est qu'après cette étape qu'ils acquièrent la capacité à synthétiser des quantités d'éthylène suffisantes pour pouvoir amorcer leur mûrissement.

En parallèle, l'amidon $\left(\mathrm{C}_{6} \mathrm{H}_{12} \mathrm{O}_{5}\right)_{n}$, se présentant sous forme de granules semi-cristallins et présent en quantités importantes dans le fruit immature, est hydrolysé progressivement en glucides solubles: saccharose, glucose et maltose impliqués dans la saveur du fruit. La progression du processus de maturation entraîne une augmentation des niveaux de sucres tels que le glucose $\mathrm{C}_{6} \mathrm{H}_{12} \mathrm{O}_{6}$. Les grandes chaînes d'amidon $(0,1$ à $200 \mu \mathrm{m})$ sont hydrolysées en de petites molécules, de l'ordre de quelques nm. Après récolte, le fruit se ramollit et l'adhésion entre les cellules devient faible.

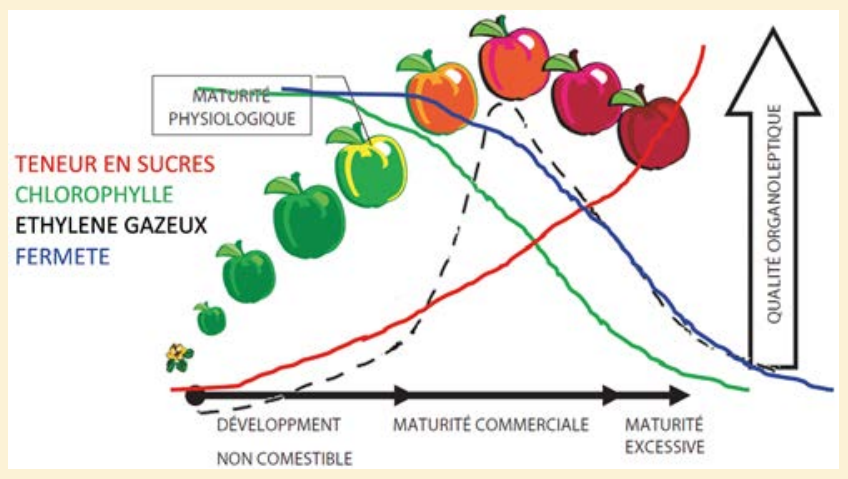

Évolution (en unités arbitraires) de paramètres physiologiques et biochimiques en fonction de la maturation des fruits climactériques. Au cours de sa vie sur l'arbre, le fruit subit une phase de croissance, puis de maturation. Durant sa période de croissance, le végétal accumule plus ou moins de substances de réserve telles que l'amidon ou les acides organiques. Ces réserves servent à assurer la continuité du métabolisme après récolte quand elles seront dégradées plus ou moins rapidement en fonction des conditions de stockage. Les différentes étapes de la vie d'un fruit précédant sa récolte jouent un rôle fondamental dans le maintien de sa qualité organoleptique durant la période de conservation ultérieure. Cette période a pour but de conserver le plus longtemps possible la qualité initiale en empêchant les dégradations du fruit. Dans cette phase, au-delà de l'état de maturité, le fruit entre en période de sénescence conduisant à sa désorganisation cellulaire et à sa mort. La maturation et la sénescence sont des étapes programmées du développement, mais peuvent être modulées par des facteurs d'environnement. 


\section{Qu'est-ce que la granularité laser (ou speckle pour les plus intimes) ?}

Le terme anglais de speckle (littéralement « tachetures », " mouchetures »), plus fréquemment employé que les termes français granularité ou tavelures, désigne une figure d'interférences aléatoires, observée lorsqu'un laser illumine un objet diffusant, en réflexion ou en transmission (figure 6).

En réflexion par une surface rugueuse, une figure de speckle provient des interférences entre les ondelettes réfléchies par les multiples petites facettes qui constituent la surface de l'objet. Le même phénomène apparaît en transmission à travers un milieu diffusant; le speckle provient alors des interférences entre toutes les ondelettes diffusées par les différents centres diffuseurs rencontrés. En un point d'interférences constructives (respectivement destructives), l'intensité est maximale (respectivement minimale). Par ailleurs, lorsque l'objet éclairé est statique, la figure de granularité ne subit pas de modification. Alors que lorsque l'objet est en mouvement, par exemple le mouvement des globules rouges dans un tissu, la figure de granularité présente des modifications dans le temps en fonction de ces mouvements.

La distribution exacte d'intensité d'une figure de speckle est impossible à écrire, car il faudrait connaître parfaitement la géométrie de l'objet diffusant à l'échelle de la longueur d'onde. Mais il est possible de décrire ses propriétés statistiques, qui sont liées aux dimensions macroscopiques de l'objet. Le calcul de la densité spectrale de puissance et la détermination de l'autocorrélation par des transformée de Fourier et transformée de Fourier inverse permet d'obtenir la taille de grain de speckle. L'évolution temporelle du speckle est également accessible en calculant le coefficient de corrélation temporelle ou au travers de la construction de la matrice THSP qui présente les variations d'intensité d'un même pixel dans une suite d'images de speckle.

L'évolution temporelle de tous ces paramètres reflète l'évolution de l'activité dans le milieu étudié. De plus, le régime de diffusion, Mie ou Rayleigh, peut être discriminé à partir du calcul de la taille de grain et du degré de polarisation de la lumière diffusée.
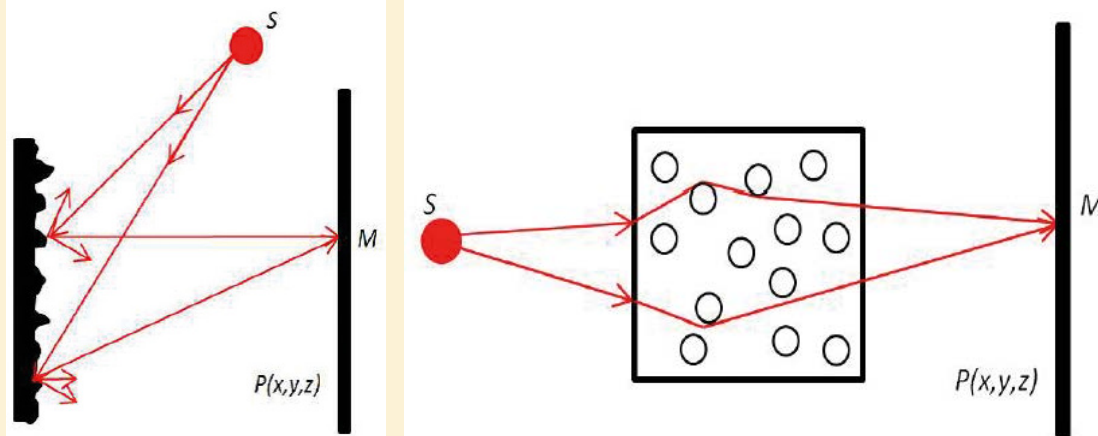

À gauche: formation en réflexion du speckle par une surface rugueuse, où $\mathrm{S}$ est la source lumineuse et $\mathrm{M}$ un point du plan d'observation P. À droite: formation en transmission du speckle par un milieu diffusant, où $\mathrm{S}$ est la source lumineuse et $\mathrm{M}$ un point du plan d'observation $P$.

\section{HคF

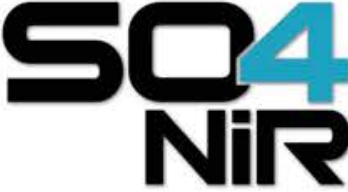

The ideal tool for aligning and characterizing optical components or optical systems in the near infrared

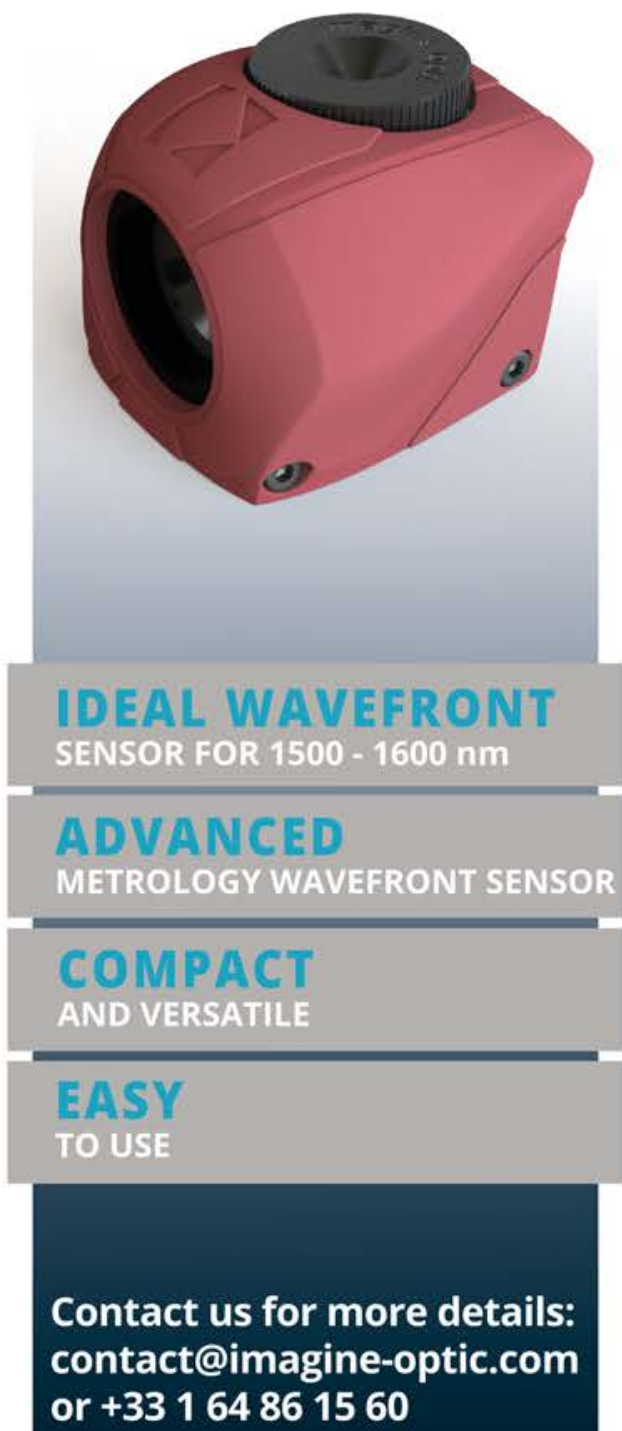

croisée $d x^{\perp}$. La tendance est la même en polarisation circulaire avec $d x^{\perp}$ Supérieur à $d x{ }^{/ /}$. Ce comportement des tailles de grain est propre aux milieux présentant un régime de diffusion de Rayleigh dominant. Les 4 courbes possèdent un même extremum au jour 8 , correspondant au pic climactérique. La décroissance de $d x$ pendant les six premiers jours peut être attribuée à la diminution de l'absorption dans le milieu puisque la teneur en chlorophylle dans les pommes diminue au cours de la maturation et atteint un stade où sa variation devient négligeable. La croissance de la taille des grains observée après le $8^{\mathrm{e}}$ jour peut quant à elle être attribuée à une augmentation de 

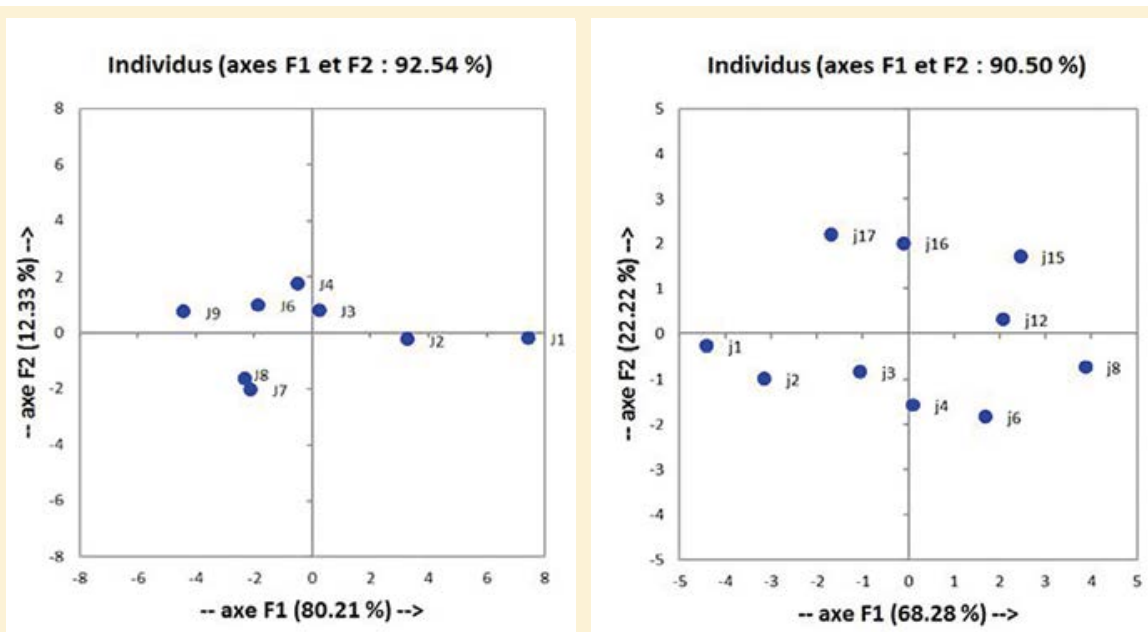

Observations groupées en fonction: du niveau de maturation des poires Conférence (à gauche); du niveau de maturation des pommes Golden (à droite).

la concentration de petits diffuseurs (tels que le glucose $\mathrm{C}_{6} \mathrm{H}_{12} \mathrm{O}_{6}$ ) dont l'effet l'emporterait sur celui de l'absorption.

Afin de mettre en évidence la corrélation entre les différents paramètres expérimentaux biochimiques et optiques et par la suite valider l'analyse du speckle comme étant une méthode optique fiable pour le suivi de la maturation des fruits, une étude statistique par analyse en composantes principales (ACP) a été menée pour chaque étude de suivi de maturation. Ce qui nous a permis de mettre en évidence qu'une corrélation positive significative existe entre la taille du grain de speckle et les taux de pigmentation, la teneur en sucres et l'intensité de fluorescence de la chlorophylle d'une part et entre le coefficient de corrélation temporelle et l'éthylène émis d'autre part. Cette étude a permis aussi de classifier les jours d'observations en fonction de leur niveau de maturation (figure 4).
D'autres études ont également été menées pour corréler les propriétés physiques des fruits à des facteurs de qualité en utilisant le speckle comme technique sans contact et non invasive. Pajuelo et al. ont testé limpact des dommages mécaniques induits dans un fruit en estimant l'activité du speckle dans le milieu [1]. Ils ont montré que la variation de l'activité, en raison des changements internes causés par des ecchymoses, est identifiée par la diminution de la corrélation entre les images de speckle. Zdunek et al. ont mesuré les changements temporels et spatiaux du speckle créé par la lumière diffusée dans les pommes en utilisant des fonctions de corrélation [2]. Ils ont montré que la corrélation entre les images de speckle diminue dans les fruits avec des vitesses différentes en fonction de leur niveau de maturité. Ils ont en outre étudié la relation entre l'activité du biospeckle d'une part, et la fermeté, la teneur en solides solubles, l'acidité titrable et la teneur en amidon d'autre part, pendant la durée de conservation de pommes. Ces résultats ont montré que les attributs de qualitéévoluent de façon significative au cours du stockage. D’autres études [3], quantifiant la variation du moment d'inertie issu de la matrice de suivi temporel (THSP) extraite des images de speckle, ont été effectuées sur des oranges. Les moments d'inertie mesurés, et montrant une dynamique de speckle qui diminue avec la baisse de qualité des fruits, ont été utilisés comme indicateurs de qualité et de sénescence ${ }^{1}$ pour les fruits. Finalement, le biospeckle a été validé comme un outil pour la détection d'agents pathogènes dans les grains de café ou de blé par Braga et al. [4].

\section{Conclusion}

L'utilisation du champ de speckle dans le suivi de la maturation des fruits permet d'explorer les paramètres de diffusion dans le fruit et d'accéder à leurs changements au cours de la maturation en s'appuyant sur des variables statiques ou dynamiques du speckle [5,6,7]. Le développement d'un prototype expérimental portable, compact et fibré permettrait à des professionnels non spécialistes de l'optique tels les ingénieurs agronomes et agroalimentaires, d'effectuer un suivi instantané et in situ de la maturation des fruits et de leur évolution physiologique interne.

En biologie, la sénescence est un processus physiologique qui entraîne une lente dégradation des fonctions de l'organisme.

\section{POUR EN SAVOIR PLUS}

[1] A. Zdunek, L. Muravsky, L. Frankevych, K. Konstankiewicz, New nondestructive method based on spatial-temporal speckle correlation technique for evaluation of apples quality during shelf-life, International Agrophysics 21, 305-310 (2007)

[2] A. Zdunek, J. Cybulska, Relation of biospeckle activity with quality attributes of apples, International Agrophysics 11, 6317-6327 (2011)

[3] G.F. Rabelo, R.A.B. Junior, I.M. Fabbro, Laser speckle techniques in quality evaluation of orange fruits, Revista Brasileira de Engenharia Agricola e Ambiental 9, 570-575 (2005)

[4] R.A. Braga, G.F. Rabelo, L.R. Granato, E.F. Santos, J.C. Machado, R. Arizaga, H.J. Rabal, M. Trivi, Detection of fungi in beans by the laser biospeckle technique, Biosystems Engineering 91, 465-469 (2005)
[5] R. Nassif, F. Pellen, C. Magné, B. Le Jeune, G. Le Brun, M. Abboud, Scattering through fruits during ripening: Laser speckle technique correlated to biochemical and fluorescence measurements, Opt. Express 20, 23887-23897 (2012)

[6] R. Nassif, Méthodes optiques cohérentes pour l'étude de milieux biologiques: utilisation des phénomènes de speckle, application à l'étude de la maturité et de la conservation des fruits, Thèse de doctorat, Université de Bretagne Occidentale et Université Saint-Joseph de Beyrouth (2013)

[7] R. Nassif, Ch. Abou Nader, C. Afif, F. Pellen, G. Le Brun, B. Le Jeune, M. Abboud, Detection of Golden apples climacteric peak by laser biospeckle measurements, Appl. Opt. 53, 8276-8282 (2014) 